\title{
Experiencia clínica en monitorización cardiaca extendida con el sistema inalámbrico satelital tipo SEEQ
}

\author{
Diego Vanegas-Cadavid $^{\mathrm{a}, \mathrm{b}, *}$, Zulma Valderrama-Barbosa ${ }^{\mathrm{a}, \mathrm{b}}$ y Linda Ibatá-Bernal ${ }^{\mathrm{b}}$
}

\author{
a Unidad de Electrofisiología Cardiovascular, Hospital Militar Central, Bogotá, Colombia \\ b Unidad de investigación, Fundarritmia - Fundación Cardiovascular, Bogotá, Colombia
}

Recibido el 13 de julio de 2017; aceptado el 4 de septiembre de 2017

Disponible en Internet el 6 de enero de 2018

\section{PALABRAS CLAVE \\ Arritmias cardíacas/ diagnóstico; \\ Electrocardiografía \\ ambulatoria}

\begin{abstract}
Resumen
Objetivos: Dar a conocer la experiencia clínica con un nuevo sistema de monitorización cardiaca extendida (por 15 días), inalámbrica y satelital en un grupo de pacientes con sospecha de arritmias cardíacas.

Metodología: Cohorte de 100 pacientes atendidos en la unidad de Electrofisiología cardiovascular de un centro de referencia, con sospecha de arritmia cardíaca, sin diagnóstico electrocardiográfico causal, a pesar de exámenes previos. Se les aplicó una monitorización cardiaca externa tipo SEEQ (Medtronic) por 15 días y se registró el desenlace.

Resultados: De un total de 100 sujetos estudiados, 51\% eran hombres, con mediana de edad de 60 años (rango: 5 - 91 años). El principal síntoma fueron las palpitaciones (42\%) y la comorbilidad más prevalente la hipertensión arterial (47\%); $98 \%$ tenían estudio de Holter previo y $46 \%$ dos estudios sin resultado conclusivo que explicara los síntomas. La monitorización tipo SEEQ documentó anormalidad electrocardiográfica significativa en $22 \%$ de los pacientes. El implante de marcapaso fue el tratamiento más aplicado y la fibrilación auricular fue la arritmia más frecuente en el $50 \%$ de los hallazgos positivos. Hubo una proporción mayor y significativa de diagnósticos positivos en el sexo masculino.

Conclusiones: La monitorización cardiaca externa inalámbrica, satelital, extendida por 15 días es una herramienta novedosa que incrementa la probabilidad de documentar una anormalidad electrocardiográfica clínicamente significativa en quienes padecen síntomas cardiovasculares recurrentes.

(C) 2017 Sociedad Colombiana de Cardiología y Cirugía Cardiovascular. Publicado por Elsevier España, S.L.U. Este es un artículo Open Access bajo la licencia CC BY-NC-ND (http:// creativecommons.org/licenses/by-nc-nd/4.0/).
\end{abstract}

\footnotetext{
* Autor para correspondencia.

Correo electrónico: diegovanegascadavid@gmail.com (D. Vanegas-Cadavid).
} 


\section{KEYWORDS}

Arrhythmias, Cardiac/diagnosis; Outpatient electrocardiography
Clinical experience in extended cardiac monitoring with the SEEQ ${ }^{\mathrm{TM}}$ satellite wireless system

\begin{abstract}
Objectives: To present the clinical experience with a new extended (for 15 days), wireless, and satellite cardiac monitoring system in a group of patients with suspicion of cardiac arrhythmia. Method: The study included a cohort of 100 patients seen in the Cardiovascular Electrophysiology Unit of a reference hospital. They were suspected of having a cardiac arrhythmia, with no electrocardiographic diagnosis of the cause, despite previous examinations. They were subjected to SEEQ-type (Medtronic) external cardiac monitoring for 15 days, with the outcomes recorded.

Results: Of the total of 100 subjects studied, $51 \%$ were male, and the median age was 60 years (range: 5 - 91 years). The main symptoms were palpitation, and the most prevalent comorbidity was arterial hypertension (47\%). Almost all (98\%) of them had a previous Holter study, and $46 \%$ had two studies, which were inconclusive in explaining the symptoms. The SEEQ monitoring recorded a significant electrocardiographic abnormality in $22 \%$ of the patients. A pacemaker implant was the treatment most applied and atrial fibrillation was the most frequent arrhythmia in $50 \%$ of the positive findings. There was a higher and significant percentage of positive diagnoses in males.

Conclusions: External, satellite, wireless cardiac monitoring extended for 15 days, is a novel tool that can increase the probability of documenting a clinically significant electrocardiographic abnormality in those patients who suffer recurrent cardiovascular symptoms.

(c) 2017 Sociedad Colombiana de Cardiología y Cirugía Cardiovascular. Published by Elsevier España, S.L.U. This is an open access article under the CC BY-NC-ND license (http:// creativecommons.org/licenses/by-nc-nd/4.0/).
\end{abstract}

\section{Introducción}

Las arritmias cardíacas son altamente prevalentes; tan solo la fibrilación auricular afecta del 1 al 4\% de la población general, e incrementa con la edad hasta en un $10 \%$ en octogenarios. Es, así mismo, causa de otras condiciones como ataque cerebrovascular y puede aparecer como consecuencia de hipertensión arterial, apnea obstructiva del sueño, obesidad o enfermedad isquémica del corazón ${ }^{1-3}$.

Esta condición agrava o propicia cambios estructurales y electrofisiológicos del corazón, y en su forma persistente o crónica produce dilatación de las cavidades atriales y/o ventriculares que lleva a falla cardiaca e incrementa el riesgo de hospitalización, embolia cerebral o muerte súbita especialmente en aquellos que padecen miocardiopatía isquémica ${ }^{1,3-8}$.

Las alteraciones del ritmo cardíaco pueden cursar asintomáticas o manifestarse a través de diferentes síntomas y signos, entre los que figuran palpitaciones, sensación de taquicardia o latidos rápidos, presíncope o síncope y adinamia súbita ${ }^{1,2}$. Estos síntomas de carácter paroxístico principalmente en su fase incipiente, más tarde pueden tornarse persistentes o crónicos. Su identificación temprana puede facilitar el diagnóstico y tratamiento oportunos, pero comúnmente es difícil su documentación.

Tradicionalmente, el electrocardiograma es considerado el "patrón de oro" ' del diagnóstico de las arritmias cardiacas aunque su obtención en el preciso momento de manifestación de los síntomas es poco probable. Para incrementar esta posibilidad se inventó la monitorización electrocardiográfica de Holter, que al extender el registro a 24 o 48 horas realza el tiempo de observación con una probabilidad mayor de capturar la arritmia.

Sin embargo, este método es útil cuando la frecuencia de presentación de los síntomas y de la arritmia es al menos interdiaria, por cuanto es menos probable que resulte en un hallazgo significativo cuando la aparición de las anomalías ocurre con intervalos de varios días o semanas.

En este estudio se pretende mostrar la experiencia primaria en Colombia de la monitorización cardiaca continua, satelital, inalámbrica, por 15 días obtenida mediante un sistema de parche electrónico SEEQ de Medtronic aplicado en pacientes que consultaron por síntomas cardiovasculares recurrentes potencialmente relacionados con arritmias cardiacas.

\section{Materiales y métodos}

\section{Tipo de estudio y población}

Se trata de un estudio retrospectivo realizado en un centro de referencia de Bogotá, en la unidad de Electrofisiología Cardiovascular, en el que se incluyó una cohorte de pacientes con sospecha de arritmia cardíaca y antecedente o no de ablación, a quienes se les realizó monitorización cardiaca satelital extendida mediante el sistema SEEQ, durante 15 días, entre el $1^{\circ}$ de abril de 2015 y el 31 de diciembre de 2016. Se excluyeron aquellos que no terminaron el seguimiento con el dispositivo o quienes al momento de concluir el estudio, no habían sido valorados por especialista para definición de conducta. 


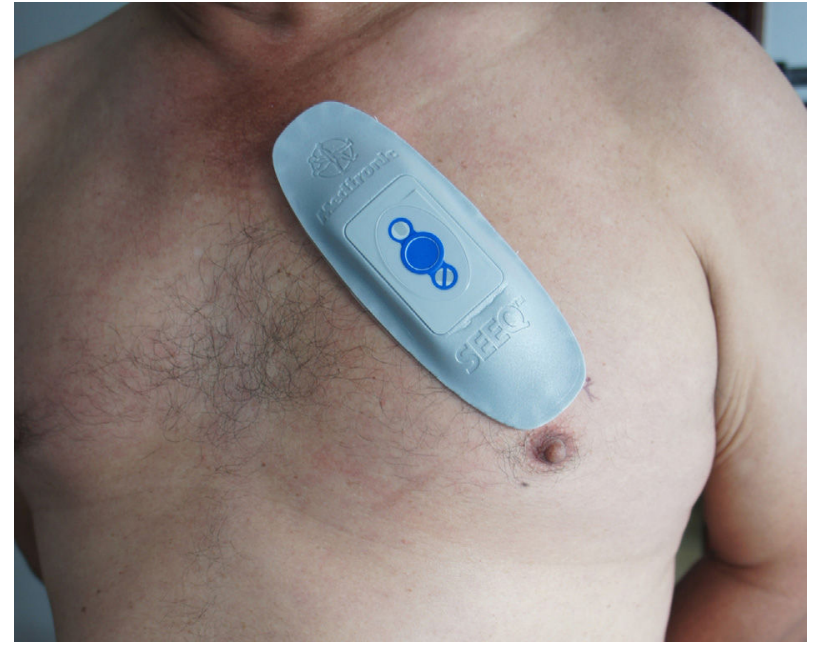

Figura 1 Dispositivo de monitorización satelital tipo SEEQ.

\section{Monitor cardíaco}

El sistema de monitorización cardiaca extendida SEEQ es un dispositivo tipo parche adhesivo, electrónico, inalámbrico, con capacidad de monitorización electrocardiográfica de un solo canal (equivalente a un DII del electrocardiograma), recientemente introducido en la práctica clínica. Este dispositivo es impermeable al agua, característica que permite su uso continuo 7,5 días cada parche. Para aumentar la probabilidad de encontrar un trazo diagnóstico y de acuerdo con estudios previos ${ }^{9}$, se hace la monitorización por 15 días utilizando secuencialmente dos parches, cada uno por 7,5 días. El parche colocado en el pecho del paciente utiliza un modem inalámbrico portátil para transmisión vía satelital, similar al uso de un teléfono celular (fig. 1). Los pacientes que recibieron esta monitorización habían sido evaluados en consulta cardiológica especializada de Electrofisiología cardiovascular por episodios recurrente de palpitaciones y/o síntomas cardiovasculares (presíncope, síncope) sin correlación electrocardiográfica a pesar del uso previo de monitorización de Holter o por tener alteraciones no conclusivas en trazos electrocardiográficos.

La recolección de la información se hizo de manera retrospectiva a partir de datos de las historias clínicas de la Unidad de Electrofisiología, de donde se tomaron las variables sociodemográficas (edad y sexo), clínicas (síntomas, antecedentes, motivo de consulta, indicación), resultados de Holter electrocardiográfico de 24 horas y conducta llevada a cabo. Los resultados de la monitorización SEEQ registrados automáticamente o activados por el paciente, fueron validados por un médico electrofisiólogo, quien fue el responsable del informe final. Se definió como evento diagnóstico significativo, una anomalía electrocardiográfica concluyente como bradicardia sinusal, diurna o en vigilia, menor de $40 \mathrm{lpm}$, sintomática, bradicardia menor de $40 \mathrm{lpm}$ asociada a ritmo de escape lento por más de 10 segundos, fibrilación auricular de al menos cinco minutos de duración, flutter atrial mayor de 5 minutos de duración, taquicardia ventricular sostenida o mayor de 30 segundos, bloqueo trifascicular de novo o paroxístico, o cualquiera de ellas asociada a síntomas; bloqueo AV de primer grado con PR mayor
Tabla 1 Información demográfica y clínica de los pacientes incluidos en el estudio

\begin{tabular}{|c|c|c|}
\hline \multicolumn{3}{|c|}{ Características } \\
\hline Edad & Años & \\
\hline Media & 56,44 & \\
\hline Mediana & 60,50 & \\
\hline Desviación estándar & 21,59 & \\
\hline Rango & $5-91$ & \\
\hline Sexo & $\mathrm{n}$ & $\%$ \\
\hline Masculino & 51 & 51,0 \\
\hline Femenino & 49 & 49,0 \\
\hline Antecedentes & $\mathrm{n}$ & $\%$ \\
\hline Hipertensión arterial & 47 & 47,0 \\
\hline Diabetes mellitus & 11 & 11,0 \\
\hline Enfermedad coronaria & 8 & 8,0 \\
\hline Obesidad & 8 & 8,0 \\
\hline Infarto de miocardio & 7 & 7,0 \\
\hline Ataque cerebrovascular & 6 & 6,0 \\
\hline Falla cardíaca & 3 & 3,0 \\
\hline Antecedente de ablación & $\mathrm{n}$ & $\%$ \\
\hline No & 85 & 85,0 \\
\hline Sí & 15 & 15,0 \\
\hline
\end{tabular}

de 320 ms o fenómeno de P en T y WPW de novo; que requirieran una conducta diferente al seguimiento clínico, bien fuera tratamiento farmacológico, implante de dispositivo de estimulación cardíaca o mapeo y ablación de la arritmia.

\section{Plan de análisis}

Toda la información obtenida de los registros médicos se recolectó en un instrumento diseñado para tal fin. Se realizó estadística descriptiva, frecuencias y proporciones para las variables categóricas y medidas de dispersión y tendencia central para las variables numéricas. Para evaluar asociación entre variables categóricas se usó la prueba de Chi cuadrado o Fisher.

\section{Resultados}

Se incluyeron 100 sujetos a quienes se les realizó monitorización cardiaca extendida satelital tipo SEEQ por 15 días. La mediana de edad fue de 60 años, con un rango entre 5 y 91 años, 51\% perteneciente al sexo masculino. Las indicaciones para la monitorización tipo SEEQ fueron: palpitaciones recurrentes sin causa clara $(27 \%)$, bradicardia $(20 \%)$, sospecha de fibrilación auricular paroxística $(21 \%)$, palpitaciones postablación (15\%), síncope $(6 \%)$, sospecha de bloqueos aurículo-ventriculares (5\%) y otras arritmias $(6 \%)$. En cuanto a antecedentes, la hipertensión arterial fue el más común, presente en aproximadamente la mitad de los pacientes. Alrededor de un $15 \%$ de la población estudiada tenía antecedente de ablación y en ellos la monitorización se realizó para seguimiento por palpitaciones (tabla 1).

Al $98 \%$ de los pacientes se les había realizado previamente al menos un estudio Holter electrocardiográfico de 24 horas. En 2\% de los casos no se solicitó, ya que los 
Tabla 2 Resultados Holter electrocardiográfico realizado previo a SEEQ

\begin{tabular}{|c|c|c|}
\hline \multicolumn{3}{|l|}{ Resultados } \\
\hline Holter No. 1 & $\mathrm{n}$ & $\%$ \\
\hline Ritmo sinusal & 41 & 41,8 \\
\hline Bradicardia sinusal & 15 & 15,3 \\
\hline Ectopias atriales o ventriculares aisladas & 14 & 14,3 \\
\hline Bloqueo $\mathrm{AV}$ de $1^{\mathrm{er}}$ o $2^{\circ}$. grado tipo 1 & 10 & 10,2 \\
\hline Taquicardia supraventricular no sostenida & 7 & 7,1 \\
\hline Fibrilación auricular paroxística & 4 & 4,1 \\
\hline Bloqueo AV de $2^{\circ}$. grado Mobitz 2 (nocturno) & 3 & 3,1 \\
\hline Taquicardia ventricular no sostenida & 3 & 3,1 \\
\hline Síndrome bradi-taqui & 1 & 1,0 \\
\hline Holter No. 2 & $\mathrm{n}$ & $\%$ \\
\hline Ritmo sinusal & 19 & 41,3 \\
\hline Ectopias atriales o ventriculares aisladas & 9 & 19,6 \\
\hline Bloqueo $\mathrm{AV}$ de $1^{\mathrm{er}}$. o $2^{\circ}$. grado tipo 1 & 6 & 13,0 \\
\hline Bradicardia sinusal & 4 & 8,7 \\
\hline Fibrilación auricular paroxística & 4 & 8,7 \\
\hline Taquicardia ventricular no sostenida & 3 & 6,5 \\
\hline Taquicardia supraventricular no sostenida & 1 & 2,2 \\
\hline
\end{tabular}

pacientes referían que la sintomatología era esporádica y no interdiaria. En el 58,2\% de los estudios efectuados, se evidenció un trazo anormal no conclusivo; entre ellos los más frecuentes fueron bradicardia sinusal no extrema y ectopias atriales o ventriculares aisladas. A $46 \%$ de los pacientes se les realizó un segundo estudio Holter electrocardiográfico de 24 horas, el cual fue normal en 41,3\% de los casos. El resultado más frecuente en este segundo examen fueron las ectopias atriales o ventriculares aisladas y los bloqueos aurículo-ventriculares de primer grado y segundo grado tipo 1 (tabla 2).

Los resultados de la monitorización se describen en la tabla 3 . Como se observa, 22 resultados se consideraron clínicamente significativos (trazos electrocardiográficos diagnósticos de una arritmia definida, que llevaron a tomar una conducta). Las arritmias más comunes fueron el flutter atrial y la fibrilación auricular en 11 pacientes, cifra correspondiente al $50 \%$ de los casos. A quienes se les hizo este diagnóstico tenían comorbilidades como hipertensión arterial $(64 \%)$, obesidad (36\%), antecedente de infarto agudo de miocardio $(27,3 \%)$, insuficiencia cardíaca $(9,1 \%)$ y ataque cerebrovascular $(9,1 \%)$.

Como se evidencia en la figura 2, la identificación del primer episodio de arritmia en el $60 \%$ de los pacientes ocurrió después del primer día de monitorización, y llegó al $90 \%$ en el día 11. A partir de los resultados obtenidos en la monitorización, 8 pacientes requirieron implante de marcapaso, 2 ablación por radiofrecuencia y los 12 restantes tratamiento farmacológico.

Al evaluar qué variables podrían relacionarse con un resultado clínicamente significativo de la monitorización SEEQ se observó asociación significativa con el sexo masculino pues en este se halló mayor proporción de diagnósticos significativos en comparación con las mujeres $(p=0,021)$.
Tabla 3 Resultados de la monitorización SEEQ y la conducta definida

\begin{tabular}{|c|c|c|}
\hline \multicolumn{3}{|l|}{ Resultados SEEQ } \\
\hline Resultados SEEQ & $\mathrm{n}$ & $\%$ \\
\hline \multicolumn{3}{|l|}{ Significativo } \\
\hline Bradicardia extrema diurna & 6 & 6,0 \\
\hline BAV de $1^{\mathrm{er}}$. grado extremo & 3 & 3,0 \\
\hline Fibrilación auricular & 7 & 7,0 \\
\hline Flutter atrial & 4 & 4,0 \\
\hline Bloqueo trifascicular & 1 & 1,0 \\
\hline WPW & 1 & 1,0 \\
\hline \multicolumn{3}{|l|}{ No significativo } \\
\hline Ritmo sinusal & 50 & 50,0 \\
\hline Bradicardia sinusal & 9 & 9,0 \\
\hline Taquicardia sinusal & 9 & 9,0 \\
\hline BAV de $1^{\mathrm{er}}$ o $2^{\circ}$. grado tipo 1 & 4 & 4,0 \\
\hline Ectopias atriales o ventriculares aisladas & 3 & 3,0 \\
\hline Episodio aislado de fibrilación auricular & 2 & 2,0 \\
\hline Taquicardia ventricular no sostenida & 1 & 1,0 \\
\hline Conducta & $\mathrm{n}$ & $\%$ \\
\hline Seguimiento clínico & 58 & 58,0 \\
\hline Tratamiento farmacológico & 23 & 23,0 \\
\hline Alta & 9 & 9,0 \\
\hline Implante de marcapasos & 8 & 8,0 \\
\hline Ablación por radiofrecuencia & 2 & 2,0 \\
\hline Resultado significativo & $\mathrm{n}$ & $\%$ \\
\hline $\mathrm{Si}$ & 22 & 22,0 \\
\hline No & 78 & 78,0 \\
\hline
\end{tabular}

$\mathrm{BAV}=$ bloqueo auriculoventricular $\mathrm{WPW}=$ síndrome de WolffParkinson-White

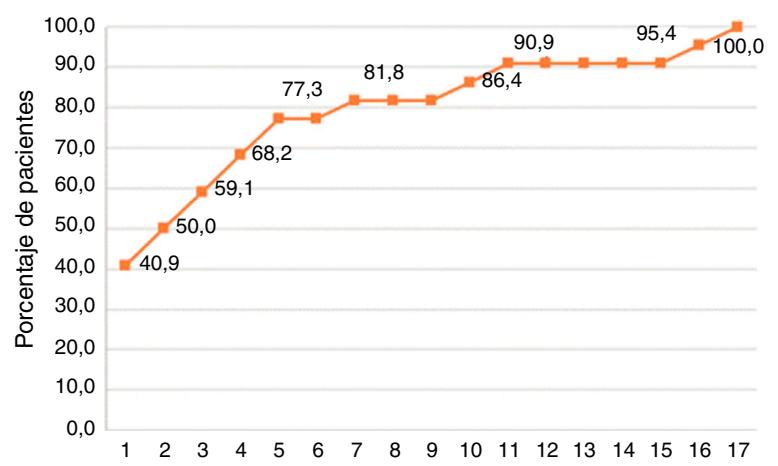

Figura 2 Registro del primer episodio arrítmico de acuerdo con el tiempo de monitorización.

No se encontraron diferencias en cuanto a edad, síntomas y antecedentes (tabla 4).

Se encontraron diferencias significativas $(p=0,0356)$ en cuanto a la indicación de la monitorización entre hombres y mujeres. En una proporción importante de hombres (31\%) la indicación de monitorización fue bradicardia comparado con $8,2 \%$ en mujeres.

Al realizar un análisis por indicación, se evidenció que la mayoría de resultados significativos pertenecían a pacientes a quienes se les realizaba monitorización por bradicardia. 
Tabla 4 Resultados monitorización SEEQ de acuerdo con características demográficas y clínicas

\begin{tabular}{|c|c|c|c|c|c|}
\hline \multirow[t]{2}{*}{ Característica } & \multicolumn{4}{|c|}{ Resultado SEEQ significativo } & \multirow[t]{2}{*}{ Valor $\mathrm{p}$} \\
\hline & No & & Sí & & \\
\hline Sexo & $\mathrm{n}$ & $\%$ & $\mathrm{~N}$ & $\%$ & 0,021 \\
\hline Femenino & 43 & $55,1 \%$ & 6 & $27,3 \%$ & \\
\hline Masculino & 35 & $44,9 \%$ & 16 & $72,7 \%$ & \\
\hline Edad & & & & & 0,334 \\
\hline Menor de 60 años & 41 & $52,6 \%$ & 9 & $40,9 \%$ & \\
\hline Mayor de 60 años & 37 & $47,4 \%$ & 13 & $59,1 \%$ & \\
\hline \multicolumn{6}{|l|}{ Síntomas } \\
\hline Palpitaciones & & & & & 0,321 \\
\hline No & 44 & $56,4 \%$ & 15 & $68,2 \%$ & \\
\hline Sí & 34 & $43,6 \%$ & 7 & $31,8 \%$ & \\
\hline Presíncope & & & & & 0,809 \\
\hline No & 62 & $79,5 \%$ & 18 & $81,8 \%$ & \\
\hline $\mathrm{Si}$ & 16 & $20,5 \%$ & 4 & $18,2 \%$ & \\
\hline Dolor torácico & & & & & 0,713 \\
\hline No & 61 & $78,2 \%$ & 18 & $81,8 \%$ & \\
\hline Sí & 17 & $21,8 \%$ & 4 & $18,2 \%$ & \\
\hline Síncope & & & & & 0,546 \\
\hline No & 63 & $80,8 \%$ & 19 & $86,4 \%$ & \\
\hline $\mathrm{Si}$ & 15 & $19,2 \%$ & 3 & $13,6 \%$ & \\
\hline Asintomático & & & & & 0,882 \\
\hline No & 75 & $96,2 \%$ & 21 & $95,5 \%$ & \\
\hline Sí & 3 & $3,8 \%$ & 1 & $4,5 \%$ & \\
\hline $\begin{array}{l}\text { Antecedente } \\
\text { de ablación }\end{array}$ & & & & & 0,120 \\
\hline No & 64 & $82,1 \%$ & 21 & $95,5 \%$ & \\
\hline Sí & 14 & $17,9 \%$ & 1 & $4,5 \%$ & \\
\hline \multicolumn{6}{|l|}{ Antecedentes } \\
\hline $\begin{array}{l}\text { Hipertensión } \\
\text { arterial }\end{array}$ & & & & & 0,750 \\
\hline No & 42 & $53,8 \%$ & 11 & $50,0 \%$ & \\
\hline Sí & 36 & $46,2 \%$ & 11 & $50,0 \%$ & \\
\hline Diabetes mellitus & & & & & 0,223 \\
\hline No & 71 & $91,0 \%$ & 18 & $81,8 \%$ & \\
\hline Sí & 7 & $9,0 \%$ & 4 & $18,2 \%$ & \\
\hline $\begin{array}{c}\text { Enfermedad } \\
\text { coronaria }\end{array}$ & & & & & 0,831 \\
\hline No & 72 & $92,3 \%$ & 20 & $90,9 \%$ & \\
\hline Sí & 6 & $7,7 \%$ & 2 & $9,1 \%$ & \\
\hline Obesidad & & & & & 0,046 \\
\hline No & 74 & $94,9 \%$ & 18 & $81,8 \%$ & \\
\hline Sí & 4 & $5,1 \%$ & 4 & $18,2 \%$ & \\
\hline $\begin{array}{l}\text { Infarto de } \\
\text { miocardio }\end{array}$ & & & & & 0,167 \\
\hline No & 74 & $94,9 \%$ & 19 & $86,4 \%$ & \\
\hline Sí & 4 & $5,1 \%$ & 3 & $13,6 \%$ & \\
\hline Falla cardiaca & & & & & 0,630 \\
\hline No & 76 & $97,4 \%$ & 21 & $95,5 \%$ & \\
\hline $\mathrm{Si}$ & 2 & $2,6 \%$ & 1 & $4,5 \%$ & \\
\hline $\begin{array}{l}\text { Ataque } \\
\text { cerebrovascula }\end{array}$ & & & & & 0,088 \\
\hline No & 75 & $96,2 \%$ & 19 & $86,4 \%$ & \\
\hline Sí & 3 & $3,8 \%$ & 3 & $13,6 \%$ & \\
\hline
\end{tabular}

" Chi cuadrado.
En total se recibieron tres alertas satelitales, sin embargo, en los tres casos, tras la valoración por especialista, se determinó que se trató de falsos positivos por artefactos.

\section{Discusión}

Los dos puntos más relevantes de la investigación fueron la utilización de un nuevo sistema inalámbrico, satelital, de monitorización cardiaca extendida por 15 días para el diagnóstico de alteraciones electrocardiográficas correlacionables con síntomas cardiovasculares, y la documentación, mediante este nuevo sistema, de una anomalía electrocardiográfica significativa, que llevó a la adopción de una nueva estrategia de tratamiento a $22 \%$ de los pacientes que no tenían diagnóstico establecido a pesar de múltiples exámenes y consultas previas. El rendimiento diagnóstico encontrado (22\%), fue superior al compararlo con un estudio realizado en una cohorte de 3.209 niños con sospecha de arritmias clínicamente significativas, donde el rendimiento de un parche con tecnología similar al SEEQ, en 14 días de seguimiento, alcanzó el $12,2 \%{ }^{10}$.

Las palpitaciones, el presíncope o el síncope cuya causa es desconocida se encuentran entre los síntomas más frecuentes por los que se consulta a médicos internistas y cardiólogos ${ }^{11}$. Tradicionalmente, la monitorización cardiaca de Holter por 24 horas es el método más utilizado para intentar capturar una arritmia cardiaca que explique esos síntomas, pero la naturaleza errática o esporádica de las mismas, conlleva un rendimiento diagnóstico bajo ${ }^{12-14}$. La monitorización cardiaca extendida mediante el sistema SEEQ es una nueva herramienta que tiene por ventajas principales su fácil portabilidad, sistema inalámbrico, resistencia al agua y capacidad para activación externa por parte del paciente y también automática, que transmite vía satelital los trazos electrocardiográficos correlacionables con síntomas.

El dispositivo se considera un avance tecnológico de gran importancia toda vez que reduce la complejidad en comparación con los otros sistemas o aparatos que suelen usarse para monitorización cardiaca (v.g. Holter de 24, 48 horas 0 7 días; monitor cardiaco externo de eventos); esto es, elimina los cables y electrodos de superficie, lo que lleva a una reducción de la interferencia eléctrica por vibración, evita la interrupción del registro por desprendimiento de un cable o electrodo, aumenta el número de días de registro, no requiere cambio diario y tolera la exposición al agua, características que redundan en comodidad para el paciente. Las desventajas principales de esta novedosa monitorización en relación con el tradicional Holter electrocardiográfico de 24 horas son la imposibilidad de realizar mediciones precisas mediante un caliper electrónico, la dependencia de un centro de recepción y la lectura a distancia, la no disponibilidad de varios canales de registro electrocardiográfico ni de valores mínimo, máximo y promedio diario y por horas, y los registros limitados de la arritmia, entre otras.

Zimetbaum et al. estudiaron la duración óptima de una monitorización cardiaca en relación con su potencial diagnóstico y afirmaron que el $90 \%$ de las arritmias cardiacas podían ser capturadas en las primeras dos semanas; una monitorización más allá de ese periodo resultaba en 


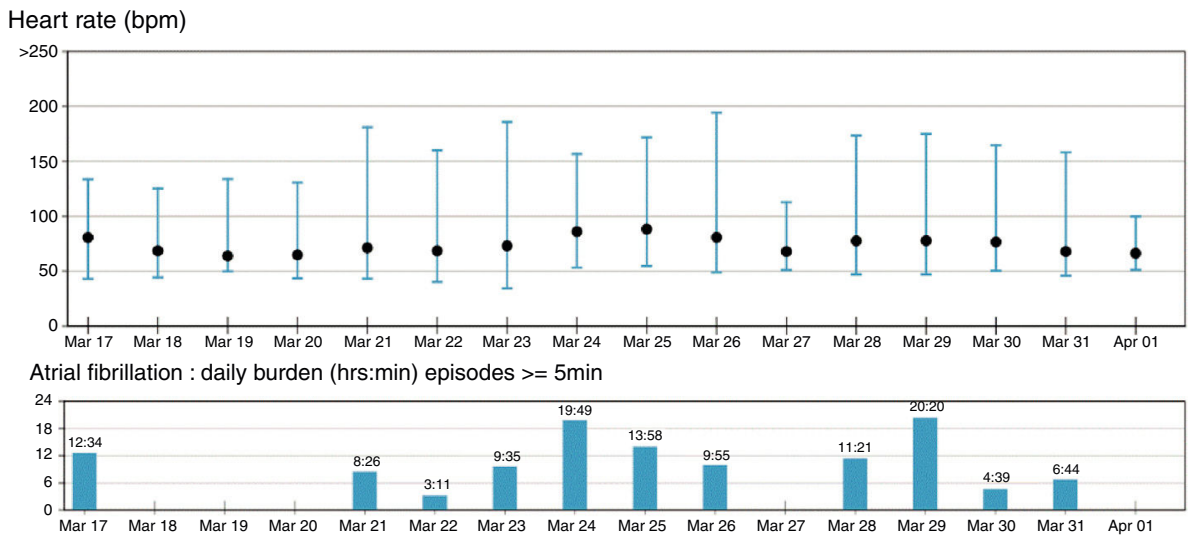

Figura 3 Resumen del registro SEEQ de 15 días de un paciente con historia de palpitaciones sin documento de arritmia a pesar de examen previo de Holter electrocardiográfico de 24 horas. Obsérvese en el histograma de barras indicativo de la carga de fibrilación auricular (episodios > de 5 minutos, frecuencia acumulada) que los días 18, 19, 20 y 27 de marzo así como el $1^{\circ}$. de abril no hubo registro de fibrilación auricular. Un Holter electrocardiográfico de 24 horas realizado esos días hubiera resultado en ausencia de diagnóstico.

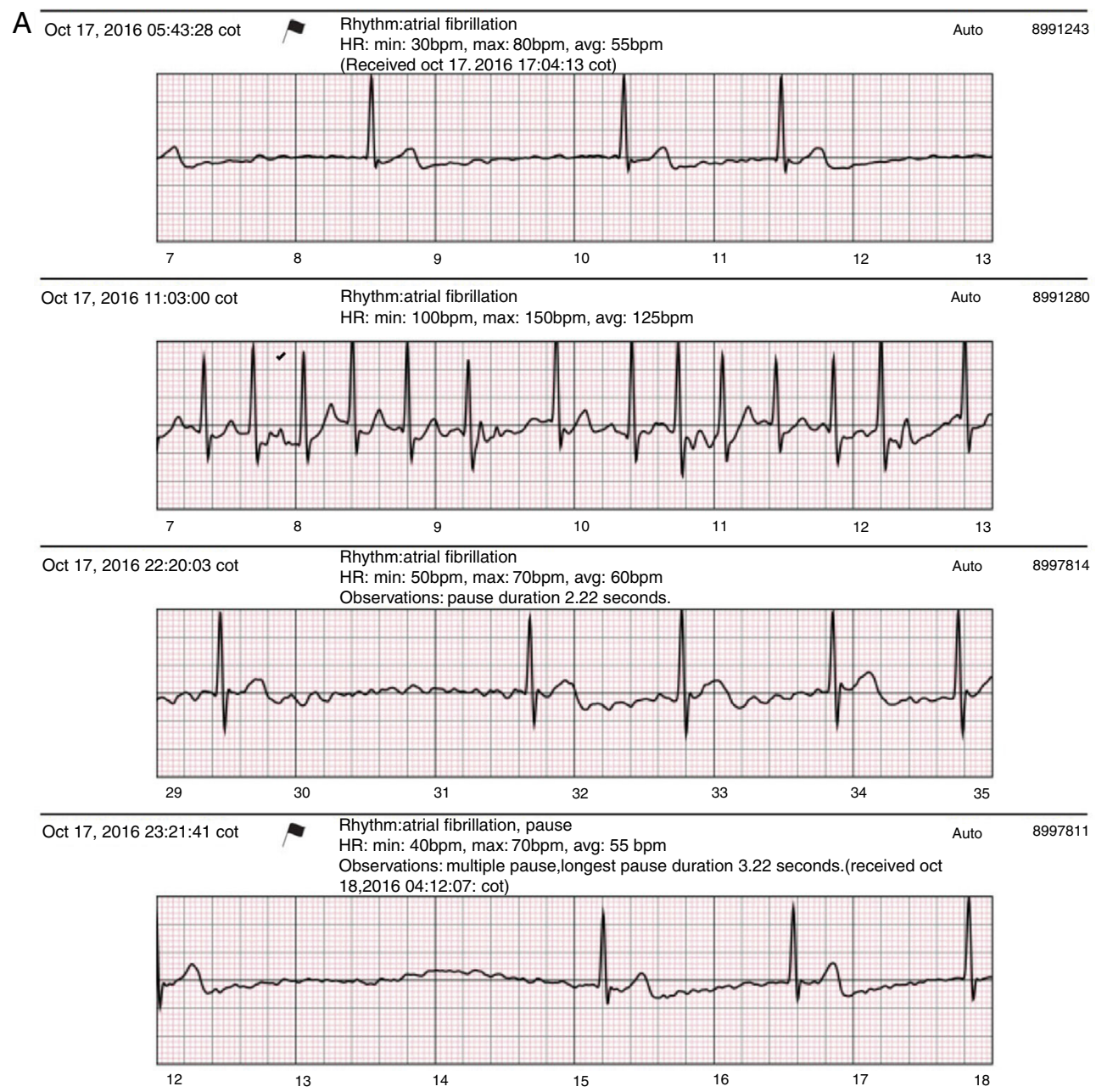

Figura 4 A: Ejemplos trazos SEEQ. Paciente con fibrilación auricular no diagnosticada previamente por monitorización de Holter, con múltiples trazos a diferentes horas de un mismo día; el signo de la bandera en el primer trazo corresponde a informe automático del sistema enviado por alerta dada la baja frecuencia cardiaca y la arritmia. B: Bradicardia sinusal extrema diurna sintomática. C: Bloqueo AV 2:1 en vigilia en paciente con historia de síncope de causa no conocida. 
Date time: Aug 05,2016 10:15:29 cot (received aug 30,2016 14:00:34 cot)

Trigger tppe: automatic

Rhythm: sinus arrhythmia, sinus bradycardia

HR: min: 30bpm, max:70bpm, avg: 50bpm,

B
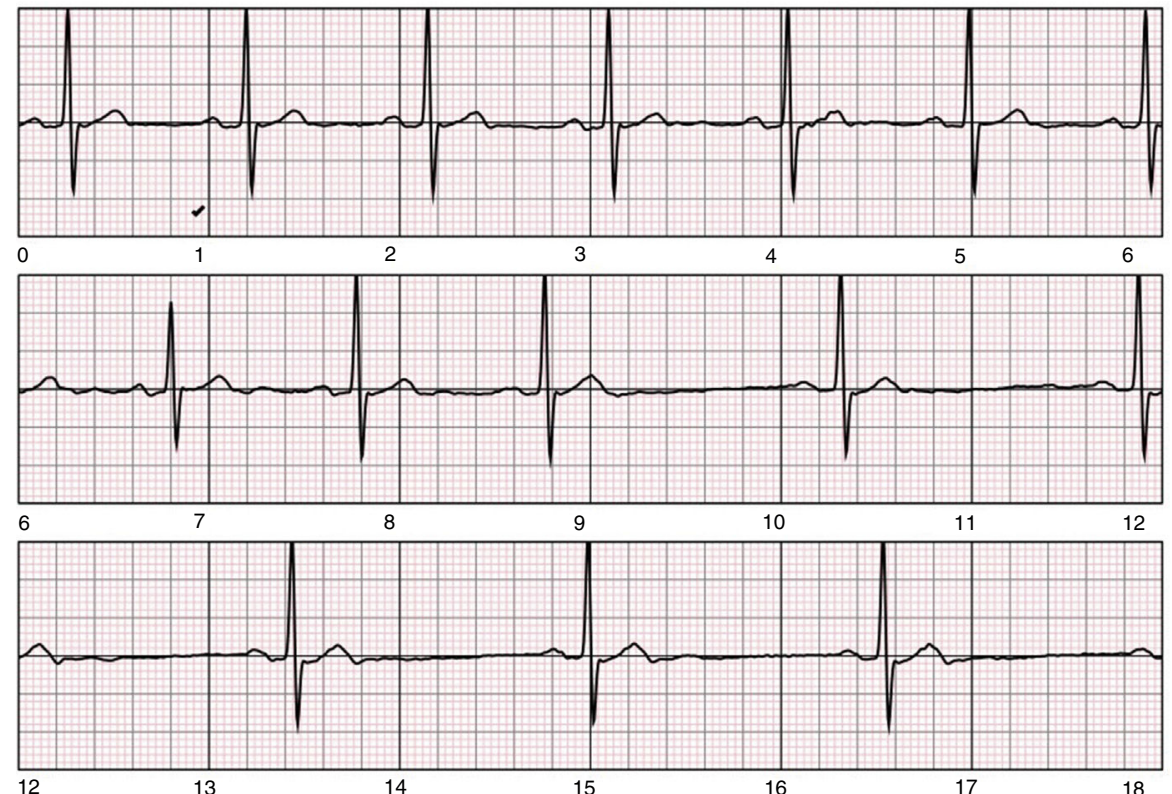

Date time: Mar 13,2016 20:32:34 cot (received mar 13,2016 20:45:13 cot)

Trigger tppe: automatic

Rhythm: 2nd degree AVB(2:1), 2nd degree AVB-mobitz I), sinus arrhythmia HR: min: 30bpm, max:80bpm, avg: 55bpm,

C
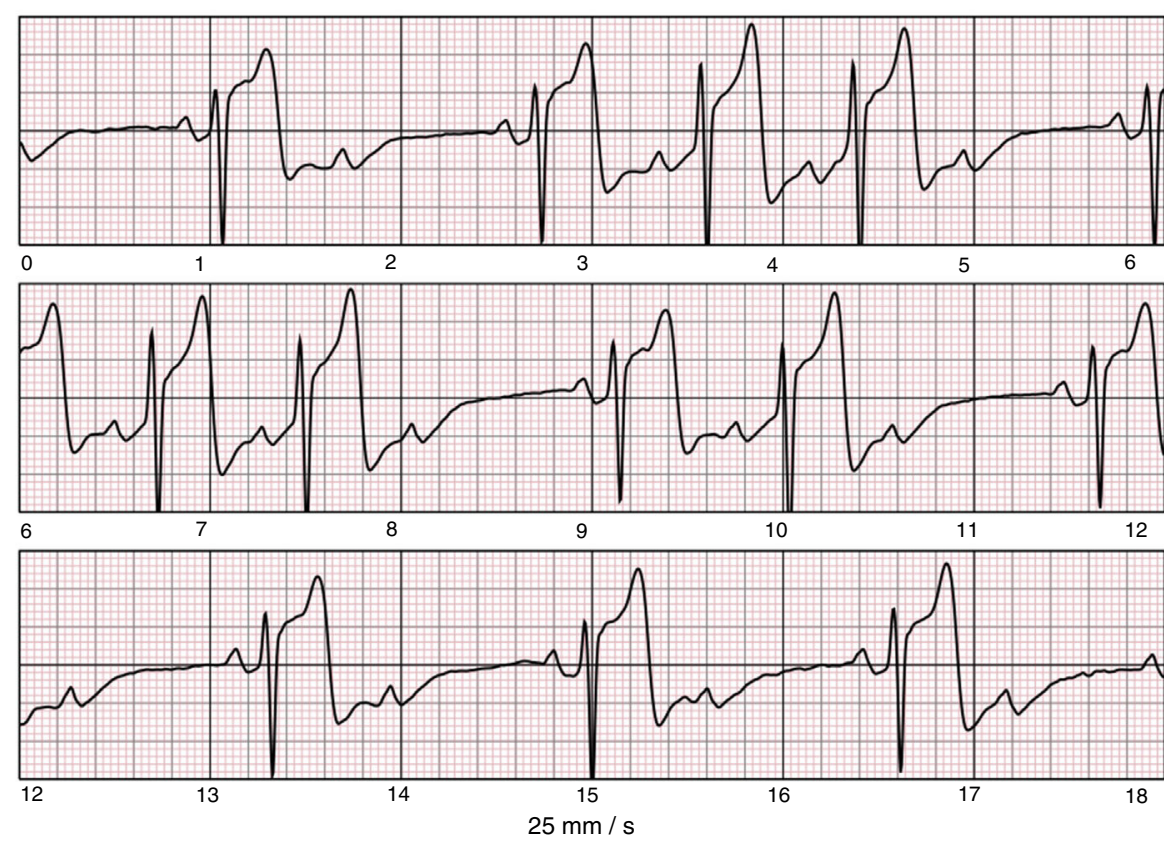

Figura 4 (continuación)

incremento del costo ${ }^{9}$. Sin embargo, además del tiempo de observación, existen otros factores que deben considerarse para incrementar el porcentaje de captura positiva de una arritmia significativa, entre otros, el tipo de síntoma, la frecuencia del mismo, el momento de la monitorización en relación con el síntoma y el tipo de población estudiada.

El tiempo de monitorización podría ser irrelevante si la frecuencia del síntoma es muy alta; esa variable cobra 
importancia cuando el síntoma es esporádico, errático o infrecuente. La monitorización de Holter de 24 o 48 horas exhibe un rendimiento diagnóstico entre 15 y $28 \%$ para síntomas de frecuencia diaria o interdiaria ${ }^{12-14}$ pero su rendimiento es muy bajo cuando el síntoma se presenta más allá de ese rango de tiempo, cuando es errático en su presentación (como sucede en la fibrilación auricular) o cuando se emplea para diagnóstico del síncope, en cuyo caso se ha reportado un rendimiento diagnóstico de tan sólo el $4 \%{ }^{15}$ (fig. 3). Los monitores cardiacos externos de "asa" fueron diseñados para extender la monitorización y realizarla específicamente durante los eventos o síntomas. Este sistema permitió incrementar el rendimiento diagnóstico de la correlación síntoma (palpitaciones intermitentes) con el documento de una arritmia hasta en el $67 \%$ tras una monitorización extendida por tres meses ${ }^{16}$; sin embargo, el dispositivo no realiza monitorización continua y requiere activación por parte del paciente durante el evento clínico, proceso que puede fallar como consecuencia del síntoma, dado que el paciente puede quedar incapacitado para realizar la activación o que no la efectúe apropiadamente, fenómeno que ha sido reportado hasta en un $23 \%$ de una serie de $\operatorname{casos}^{17}$; adicionalmente, los electrodos de superficie y cables del monitor producen incomodidad después de cierto número de días de uso, hecho que limita la complacencia por parte del paciente $y$, finalmente algunos de estos dispositivos no registran los eventos asintomáticos lo cual es de trascendental importancia en el caso de arritmias cardiacas como la fibrilación auricular (30\% de los eventos en pacientes hipertensos y hasta $50 \%$ en otras series $)^{1,18}$. De acuerdo con Balmelli et al., el monitor cardiaco de eventos debe disponer de activación automática pues un porcentaje de hallazgos electrocardiográficos significativos son capturados mediante ese algoritmo y no por activación del paciente ${ }^{18}$. Por otra parte, la monitorización cardiaca implantable ha demostrado que para síntomas esporádicos, erráticos o aislados (periodicidad de aparición de al menos un mes) se requiere un tiempo de monitorización prolongado para la captura de una anomalía, usualmente mayor a un año ${ }^{19}$.

Estudios recientes que utilizan monitorización cardiaca extendida en poblaciones de pacientes con sintomatología similar a la presentada en esta serie, han arrojado resultados concluyentes correlacionables con arritmias clínicamente significativas hasta en el $41 \%{ }^{20}$. No obstante, la población sujeto de esa investigación presentaba una frecuencia mayor de síntomas, una incidencia de enfermedad cardiovascular más alta, con enfermedad coronaria del $21 \%$, que contrasta con el $15 \%$ de la serie actual, aunado a que la monitorización cardiaca fue extendida por 30 días. El rendimiento diagnóstico en el estudio de Rothman et al. ${ }^{20}$ fue incluso más alto cuando el síntoma investigado fue presíncope o síncope recurrente $(89 \%)$, pese a la frecuencia precedente del síntoma extremadamente alta en ese subgrupo de pacientes (1-99 eventos presincopales y/o 1-10 eventos sincopales antes de la monitorización). La hipertensión arterial fue la comorbilidad más común en ambos estudios, con un porcentaje similar, $47 \%$ en la presente serie versus $52 \%$ en el estudio en mención. La hipertensión arterial es la enfermedad más relacionada con fibrilación auricular; su padecimiento incrementa el riesgo de esa arritmia en 70 a 80\%, y es la causa de aproximadamente el $20 \%$ de los casos de fibrilación auricular en el mundo occidental ${ }^{1}$. Esa relación puede explicar la presencia de esa arritmia o sucedáneas (flutter atrial) en 11 de los 22 resultados positivos encontrados en la presente investigación (fig. 4A) y así mismo en el estudio llevado a cabo por Rothman et al. en el que también fue la arritmia más común (23\%). Se evidenció que los pacientes con hallazgos positivos de fibrilación y/o flutter atrial en la monitorización SEEQ de la presente investigación tenían una tasa alta de comorbilidades, además de hipertensión, obesidad y enfermedad coronaria.

Los hallazgos no conclusivos del primer Holter y los resultados finales conclusivos del SEEQ sugieren que los pacientes con síntomas cardiovasculares que tienen sospecha de origen arrítmico podrían beneficiarse en mayor proporción de una monitorización extendida y no de la repetición de una nueva monitorización Holter ${ }^{21}$. La observación prolongada de los síntomas y la monitorización concomitante amplían la posibilidad de establecer una correlación clínica significativa. En esta serie se requirieron tres días para el registro del $50 \%$ de los hallazgos positivos, cinco a seis días para la obtención del $70 \%$ de los hallazgos y diez a once días para la obtención del $90 \%$ de los resultados positivos (fig. 2).

De igual forma, el porcentaje de hallazgos positivos del $22 \%$ obtenido mediante la monitorización SEEQ en la presente serie de casos, podría incrementarse si se consideran candidatos a esta monitorización aquellos con palpitaciones recurrentes, cuya sintomatología sea expresada por el paciente de manera más consistente en la duración de los eventos, por ejemplo de al menos 1 minuto de duración, cuando los eventos sean de mayor frecuencia, al menos semanal y cuando el número total de eventos acumulados sea más alto, similar al estudio de Rothman ${ }^{20}$. No es suficiente la mera descripción del síntoma; se requiere retomar las características semiológicas del mismo para realzar la posibilidad de documentar su correlato electrocardiográfico.

El hallazgo de bradicardia diurna o en vigilia, significativa (<40 lpm) (más frecuente entre los hombres) y la posibilidad de que se reconozca como explicativa de los síntomas conducentes al implante de un marcapaso, han sido reconocidos por otros estudios de monitorización cardiaca implantable ${ }^{19}$. Los pacientes que exhiben bradicardia en la monitorización de Holter, no significativa o aislada inicialmente, que persisten con progresión a eventos diurnos en estudios de mayor duración (monitorización SEEQ o monitor implantable) pueden requerir el implante de un dispositivo en un porcentaje tan alto como del $70 \%$ (figs. $4 \mathrm{~B}$ y C) ${ }^{19}$.

Dentro de las limitaciones del estudio se encuentran aquellas inherentes al diseño del mismo. Al ser observacional, los resultados reflejan lo que ocurre en el mundo real. La población estudiada es heterogénea tanto en edad como en características clínicas, hecho que afecta los resultados pues la probabilidad de esta población de padecer una arritmia es diferente a la de las poblaciones de estudios experimentales con criterios de inclusión más estrictos. Sin embargo, es una aproximación a lo que ocurre en la práctica cotidiana y por ende puede brindar información sustancial acerca de la utilidad de este método diagnóstico, así como elementos de juicio para la elección de la población que más se beneficiaría con su uso. 


\section{Responsabilidades éticas}

Protección de personas y animales. Los autores declaran que para esta investigación no se han realizado experimentos en seres humanos ni en animales.

Confidencialidad de los datos. Los autores declaran que han seguido los protocolos de su centro de trabajo sobre la publicación de datos de pacientes.

Derecho a la privacidad y consentimiento informado. Los autores declaran que en este artículo no aparecen datos de pacientes.

\section{Financiación}

Este estudio fue patrocinado por la Fundarritmia - Fundación Cardiovascular.

\section{Conflicto de intereses}

Ninguno.

\section{Bibliografía}

1. McLeod C, Gersh B. The epidemiology of atrial fibrillation. Chapter 1. En: Shenasa M, Camm AJ, editores. Management of Atrial Fibrillation. A Practical Approach. Oxford University Press; 2015. p. 1-3.

2. Rahman F, Benjamin E. Classification and Epidemiology of Atrial Fibrillation. Chapter 1 Atrial Fibrillation. En: Waldo A, editor. A Multidisciplinary Approach to Improving Patient Outcomes. Estes III N. A. M. Cardiotext Publishing; 2015. p. 1-7.

3. Chugh S, Havmoeller R, Narayanan K, Singh D, Rienstra M, Benjamin EJ, et al. Worldwide epidemiology of atrial fibrillation: A global burden of disease 2010 study. Circulation. 2014;129:837-47.

4. Wijffels M, Kirchhof C, Dorland R, Allessie M. Atrial fibrillation begets atrial fibrillation. A study in awake chronically instrumented goats. Circulation. 1995;92:1954-68.

5. Anne W, Willems R, Holemans P, Beckers F, Roskams T, Lenaerts I, et al. Self-terminating AF depends on electrical remodeling while persistent AF depends on additional structural changes in a rapid atrially paced sheep model. J Molec Cell Cardiol. 2007;43:148-58.

6. Sanfilippo A, Abascal V, Sheehan M, Oertel LB, Harrigan P, Hughes RA, et al. Atrial enlargement as a consequence of atrial fibrillation. A prospective echocardiographic study. Circulation. 1990;82:792-7.

7. Platonov P, Mitrofanova L, Orshanskaya V, Ho SY. Structural abnormalities in atrial walls are associated with presence and persistency of atrial fibrillation but not with age. J Am Coll Cardiol. 2011;58:2225-32.
8. Abed H, Samuel C, Lau D, Kelly DJ, Royce SG, Alasady M, et al. Obesity results in progressive atrial structural and electrical remodeling: implications for atrial fibrillation. Heart Rhythm. 2013;10:90-100.

9. Zimetbaum P, Kim K, Josephson M, Goldberger A, Cohen D. Diagnostic yield and optimal duration of continuous-loop event monitoring for the diagnosis of palpitations. A costeffectiveness analysis. Ann Intern Med. 1998;128:890-5.

10. Bolourchi M, Batra A. Diagnostic yield of patch ambulatory electrocardiogram monitoring in children (from a national registry). Am J Cardiol. 2015;115:630-4.

11. Kroenke K, Arrington M, Mangelsdorff A. The prevalence of symptoms in medical outpatients and the adequacy of therapy. Arch Intern Med. 1990;150:1685-9.

12. Bass E, Curtiss E, Arena V, Hanusa B, Cecchetti A, Karpf M, et al. The duration of Holter monitoring in patients with syncope. Is 24 hours enough? Arch Int Med. 1990;150:1073-8.

13. Gibson T, Heitzman M. Diagnostic efficacy of 24-hour electrocardiographic monitoring for syncope. Am J Cardiol. 1984;53:1013-7.

14. Krahn A, Renner S, Klein G, Yee R, Skanes A, Evans EM. The utility of Holter monitoring compared to loop recorders in the evaluation of syncope and presyncope. Ann Noninvasive Electrocardiol. 2000;5:284-9.

15. Barbeito-Caamaño C, Sánchez-Fernández G, Bouzas-Mosquera A, Broullón F, Álvarez-García N, Vázquez-Rodríguez JM. Diagnostic and prognostic efficiency of 24-hour Holter monitoring in patients with syncope. Medicina Clínica (English Edition). 2016;147:148-50.

16. Kinlay S, Leitch J, Neil A, Chapman B, Hardy D, Fletcher P. Cardiac event recorders yield more diagnoses and are more cost-effective than 48-hour Holter monitoring in patients with palpitations: A controlled clinical trial. Ann Intern Med. 1996;124:16-20.

17. Sivakumaran S, Krahn A, Klein G, Finan J, Yee R, Renner S, Skanes A. A prospective randomized comparison of loop recorders versus Holter monitors in patients with syncope or presyncope. Am J Med. 2003;115:1-5.

18. Balmelli N, Naegeli B, Bertel O. Diagnostic yield of automatic and patient-triggered ambulatory cardiac event recording in the evaluation of patients with palpitations, dizziness, or syncope. Clin Cardiol. 2003;26:173-6.

19. Vanegas $D$, Jiménez $N$, Rincón $C$, Hernández $M$, Valderrama $Z$. Experiencia clínica con el uso del monitor cardiaco implantable. Rev Colomb Cardiol. 2016, http://dx.doi.org/10.1016/ j.rccar.2016.08.004.

20. Rothman S, Laughlin J, Seltzer J, Walia J, Baman R, Siouffi S, et al. The Diagnosis of Cardiac Arrhythmias: A prospective multicenter randomized study comparing mobile cardiac outpatient telemetry versus standard loop event monitoring. J Cardiovasc Electrophysiol. 2007;18:1-7.

21. Rockx M, Hoch J, Klein G, Yee R, Skanes A, Gula L, Krahn A. Is ambulatory monitoring for "community-acquired" syncope economically attractive? A cost-effectiveness analysis of a randomized trial of external loop recorders versus Holter monitoring. Am Heart J. 2005;150:e1, 1065-e5.1075. 